\title{
Pulse Energy and Pulse Duration Effects in the Ionization and Fragmentation of Iodomethane by Ultraintense Hard X Rays
}

\author{
X. Li, ${ }^{1}$ L. Inhester, ${ }^{2,3}$ S. J. Robatjazi, ${ }^{1}$ B. Erk ${ }^{4}$ R. Boll,${ }^{5,6}$ K. Hanasaki, ${ }^{2,3}$ K. Toyota, ${ }^{2,3}$ Y. Hao, ${ }^{2,3,7}$ C. Bomme, ${ }^{4}$ \\ B. Rudek,${ }^{8}$ L. Foucar, ${ }^{9}$ S. H. Southworth, ${ }^{10}$ C. S. Lehmann, ${ }^{10,11}$ B. Kraessig, ${ }^{10}$ T. Marchenko, ${ }^{12}$ \\ M. Simon, ${ }^{12}$ K. Ueda, ${ }^{13}$ K. R. Ferguson, ${ }^{14}$ M. Bucher, ${ }^{10,14}$ T. Gorkhover, ${ }^{14,15}$ S. Carron, ${ }^{14}$ R. Alonso-Mori, ${ }^{14}$ \\ J. E. Koglin, ${ }^{14}$ J. Correa, ${ }^{2,4}$ G. J. Williams, ${ }^{14,16}$ S. Boutet,${ }^{14}$ L. Young, ${ }^{10,17}$ C. Bostedt, ${ }^{10,18,19}$ \\ S.-K. Son, ${ }^{2,3}$ R. Santra, ${ }^{2,3,20}$ D. Rolles, ${ }^{1,4}$ and A. Rudenko ${ }^{1}$ \\ ${ }^{1}$ J. R. Macdonald Laboratory, Department of Physics, Kansas State University, Manhattan, Kansas, USA \\ ${ }^{2}$ Center for Free-Electron Laser Science, DESY, Hamburg, Germany \\ ${ }^{3}$ The Hamburg Centre for Ultrafast Imaging, Hamburg, Germany \\ ${ }^{4}$ Deutsches Elektronen-Synchrotron (DESY), Hamburg, Germany \\ ${ }^{5}$ Max Planck Institute for Nuclear Physics, Heidelberg, Germany \\ ${ }^{6}$ European XFEL, Schenefeld, Germany \\ ${ }^{7}$ Institute of Theoretical Physics and Department of Physics, University of Science and Technology Beijing, \\ Beijing, People's Republic of China \\ ${ }^{8}$ Physikalisch-Technische Bundesanstalt (PTB), Braunschweig, Germany \\ ${ }^{9}$ Max Planck Institute for Medical Research, Heidelberg, Germany \\ ${ }^{10}$ Chemical Sciences and Engineering Division, Argonne National Laboratory, Lemont, Illinois, USA \\ ${ }^{11}$ Fachbereich Chemie, Philipps-Universität Marburg, Marburg, Germany \\ ${ }^{12}$ Sorbonne Université, CNRS, Laboratoire de Chimie Physique-Matière et Rayonnement, LCPMR, Paris, France \\ ${ }^{13}$ Institute of Multidisciplinary Research for Advanced Materials, Tohoku University, Sendai, Japan \\ ${ }^{14}$ LCLS, SLAC National Accelerator Laboratory, Menlo Park, California, USA \\ ${ }^{15}$ Institut für Optik und Atomare Physik, Technische Universität Berlin, Berlin, Germany \\ ${ }^{16}$ NSLS-II, Brookhaven National Laboratory, Upton New York, USA \\ ${ }^{17}$ Department of Physics and James Franck Institute, The University of Chicago, Chicago, Illinois, USA \\ ${ }^{18}$ Paul Scherrer Institut, Villigen-PSI, Villigen, Switzerland \\ ${ }^{19}$ Institute of Chemical Sciences and Engineering, Ecole Polytechnique Fédérale de Lausanne, Lausanne, Switzerland \\ ${ }^{20}$ Department of Physics, Universität Hamburg, Hamburg, Germany
}

(Received 20 June 2019; revised 24 January 2021; accepted 2 July 2021; published 27 August 2021)

\begin{abstract}
The interaction of intense femtosecond $\mathrm{x}$-ray pulses with molecules sensitively depends on the interplay between multiple photoabsorptions, Auger decay, charge rearrangement, and nuclear motion. Here, we report on a combined experimental and theoretical study of the ionization and fragmentation of iodomethane $\left(\mathrm{CH}_{3} \mathrm{I}\right)$ by ultraintense $\left(\sim 10^{19} \mathrm{~W} / \mathrm{cm}^{2}\right)$ x-ray pulses at $8.3 \mathrm{keV}$, demonstrating how these dynamics depend on the x-ray pulse energy and duration. We show that the timing of multiple ionization steps leading to a particular reaction product and, thus, the product's final kinetic energy, is determined by the pulse duration rather than the pulse energy or intensity. While the overall degree of ionization is mainly defined by the pulse energy, our measurement reveals that the yield of the fragments with the highest charge states is enhanced for short pulse durations, in contrast to earlier observations for atoms and small molecules in the soft x-ray domain. We attribute this effect to a decreased charge transfer efficiency at larger internuclear separations, which are reached during longer pulses.
\end{abstract}

DOI: 10.1103/PhysRevLett.127.093202

Ultraintense femtosecond pulses generated by $\mathrm{x}$-ray free-electron lasers (XFELs), together with advanced imaging and spectroscopic techniques, have demonstrated an enormous potential for revealing material structures and ultrafast dynamics with an unprecedented combination of spatial and temporal resolution [1-3]. In particular, the ability to produce pulses with more than $10^{12} \mathrm{x}$-ray photons with a duration of only a few tens of femtoseconds provides the basis for the concept of "diffraction before destruction" [4], where a high-resolution diffraction image reflecting the intact sample structure can be obtained before the latter is significantly altered by the absorbed $\mathrm{x}$ rays. The realization of this idea has led to the development of serial femtosecond nanocrystallography $[3,5]$ and has potential for diffractive imaging of single macromolecules $[3,6]$.

However, since the probability for $\mathrm{x}$-ray absorption is typically much higher than for elastic scattering, the intense $\mathrm{x}$-ray pulses can change the electronic structure and nuclear 
geometry of the sample even on such ultrafast, femtosecond timescales. Therefore, a quantitative understanding of how the electrons in the individual atoms respond to (multiple) x-ray absorption [7-11], by how much the distances between the atoms change during the $\mathrm{x}$-ray pulse [12-16], and how the absorbed x-ray energy and the created charge are redistributed within the sample [13-23], is crucial for the choice of XFEL parameters, feasibility estimates, and the interpretation of the results of imaging studies, as well as many other XFEL applications. This understanding is also of fundamental interest in atomic and molecular physics as a prototypical example of highly nonlinear reactions involving correlated electronic and nuclear motion.

Being one of the best-studied polyatomic systems in ultrafast photochemistry, the iodomethane $\left(\mathrm{CH}_{3} \mathrm{I}\right)$ molecule was recently used as a representative model case for studying the dynamics of multiple photoabsorption, Auger decay, charge transfer, and nuclear motion induced by soft x-ray pulses [17-21], as well as by moderately intense [15] and ultraintense [16] hard x-ray pulses. Besides its well-known spectroscopic properties, $\mathrm{CH}_{3} \mathrm{I}$ was chosen for these studies because of the very high degree of localization of the x-ray absorption at the iodine atom, which makes it a model case for the site-specific interaction of $\mathrm{x}$ rays with heteronuclear molecules. For moderately intense pulses, efficient charge rearrangement results in a reduction of the final charge state of the absorbing heavy atom, whereas it does not considerably affect the total charge state of the molecule [13-15]. At higher intensities, however, the electron transfer to the heavy atom was shown to increase the total charge state of the ionized system - an effect dubbed "charge-rearrangement-enhanced x-ray ionization of molecules" (CREXIM) $[16,24]$. The dynamics in the vicinity of a high- $Z$ element within a polyatomic system was also found to be important for XFEL imaging applications, where the presence of heavy atoms in the sample increases local radiation damage $[25,26]$ and has been proposed as a way of phasing the diffraction data $[27,28]$.

In this Letter, we show how the ionization and fragmentation of iodomethane by ultraintense $(0.5-2.2 \times$ $10^{19} \mathrm{~W} / \mathrm{cm}^{2}$ ) hard $\mathrm{x}$ rays at $8.3 \mathrm{keV}$ photon energy ( $\sim 1.5 \AA$ wavelength) and, in particular, the ensuing charge rearrangement dynamics depend on the pulse energy and pulse duration. We demonstrate that the timing of ionization steps leading to a particular reaction product and, thus, the product's final kinetic energy, is determined by the pulse duration rather than the pulse energy or intensity. While the overall degree of ionization is mainly defined by the pulse energy, our experimental results show that both the total charge state of the molecule and the yield of the highly charged heavy atom fragments are enhanced for shorter pulses. This observation is in stark contrast with earlier findings for $\mathrm{Ne}$ [7] and $\mathrm{Ar}$ [10] atoms, and $\mathrm{N}_{2}$ molecules [12,29], where a shorter pulse resulted in significantly reduced ionization.

The experiment was performed at the Coherent X-Ray Imaging (CXI) beamline [30] of the Linac Coherent Light Source (LCLS). Femtosecond X-ray pulses at a photon energy of $8.3 \mathrm{keV}$ were focused to a focal area of about $0.1 \mu \mathrm{m}^{2}$ in the center of the CXI vacuum chamber hosting an ion momentum imaging spectrometer. The $\mathrm{x}$-ray beam crossed a collimated pulsed beam of cold iodomethane molecules. The created ionic fragments were projected onto a time- and position-sensitive detector by a homogeneous electric field. From the measured ion times of flight and hit positions on the detector, the ion charge state and 3D momentum distributions were calculated.

To study the pulse energy and pulse duration dependence, the experiment was repeated at varying pulse energies while keeping the pulse duration fixed, and at varying pulse durations while keeping the pulse energy fixed. The pulse energy was changed by introducing a set of silicon foils of varying thicknesses upstream of the CXI instrument. Three narrow pulse energy windows centered at $0.25,0.74$, and $1.19 \mathrm{~mJ}$ were used. The pulse duration was altered by adjusting the length of the electron bunch. Three different settings with nominal values of the $\mathrm{x}$-ray pulse duration of 20, 30, and $60 \mathrm{fs}$ were used. Details of the pulse energy selection and pulse duration determination are described in the Supplemental Material (SM) [31]. All experiments were simulated with the XMOLECULE toolkit [33], extended to include $a b$ initio photoionization cross sections and decay rates for all the various electronic hole configurations created during the subsequent ionization steps [24]. To account for the spatial distribution of the x-ray fluence in the interaction region, the simulation results were integrated over the spatial profile of the pulse, which was determined using the measured charge state distributions (CSDs) of argon atoms as described in Ref. [34]. Further details of the experimental setup and simulation procedures are described in the Methods section of Ref. [16].

Overall, the ionization of $\mathrm{CH}_{3} \mathrm{I}$ by intense hard $\mathrm{x}$ rays can be described by an intuitive sequential model that involves a series of single-photon absorption steps $[15,16]$. This model predicts that the degree of ionization increases with the number of photons in the pulse, which is confirmed by our experimental data. Supplemental Material Fig. S2 [31] shows that for high pulse energies, the CSDs extend to higher charge states, and the highly charged ions are more abundant. With increasing pulse energy at a fixed pulse duration, the ion fragments can be expected to reach higher average kinetic energies since a larger photoabsorption rate (corresponding to smaller time intervals between ionizations) results in a given ionization step occurring at shorter internuclear distances, thus leading to stronger Coulomb repulsion [12]. However, rather counterintuitively, the measured iodine ion kinetic energy distributions plotted in Fig. 1(a) show no dependence on the x-ray pulse energy. 
This trend is also predicted by the outcome of the XMOLECULE simulation, as shown in Supplemental Material Fig. S3(a) [31]. The almost identical kinetic energy distributions for a given charge state observed at different pulse energies imply that these charge states were produced, on average, at similar internuclear separations and, thus, with similar ionization timing. This surprising finding can be explained by the following qualitative picture: Since the photoabsorption probability increases with the intensity, a specific charge state $n$ is, on average, reached faster for the higher-energy pulse. However, if this charge state is not yet the highest charge state reachable at this photon energy, the molecule will likely be further ionized during the remainder of the pulse. Therefore, such a charge state $n$ can survive and be detected only if the last ionization step leading to the creation of this charge state occurs close to the end of the pulse, when the probability for subsequent ionization to a charge state higher than $n$ is low. This implies that the total time it takes to reach such a charge state $n$ can be approximated by the pulse duration. Since, on average, a fixed number of photons $N_{\mathrm{ph}}$ needs to be absorbed to reach a particular charge state $n$, the average time interval between the individual photoabsorption steps is determined by the pulse duration divided by $N_{\mathrm{ph}}$, and does not depend on the pulse energy. This intuitive picture is corroborated by Fig. S4, where the simulated average time to reach a final charge state of $\mathrm{I}^{20+}$ is plotted for different fluences and different pulse durations, and is found to be defined solely by the latter. Assuming that the photoabsorption cross section is constant for all iodine charge states (which is a reasonable approximation for our experimental conditions), one can rigorously show that for a given number of absorbed photons, the average time interval between subsequent photoabsorption steps is independent of the pulse energy and fluence and is proportional to the pulse duration [35].

The above reasoning readily explains the independence of the kinetic energy distributions on the pulse energy observed in Fig 1(a). Because the pulse duration is the same for the three cases, the corresponding kinetic energy distributions are almost identical even though the pulses have different pulse energies, fluences, and intensities. In contrast, varying the pulse duration while keeping the pulse energy constant [see Fig. 1(b)] results in significant changes of the kinetic energy distributions of iodine ions. We find that for charge states above $\mathrm{I}^{9+}$, which are predominantly created by absorption of more than one photon, shorter pulses produce higher-energy iodine ions, reflecting the fact that, on average, it takes less time in a shorter pulse to reach a given fragment charge state. This trend is also predicted by the simulations shown in Fig. S3(b).

Since the change in the pulse duration affects the average ionization timing, and hence the internuclear distance at which a particular charge state is reached, the charge rearrangement dynamics should also be affected. This is

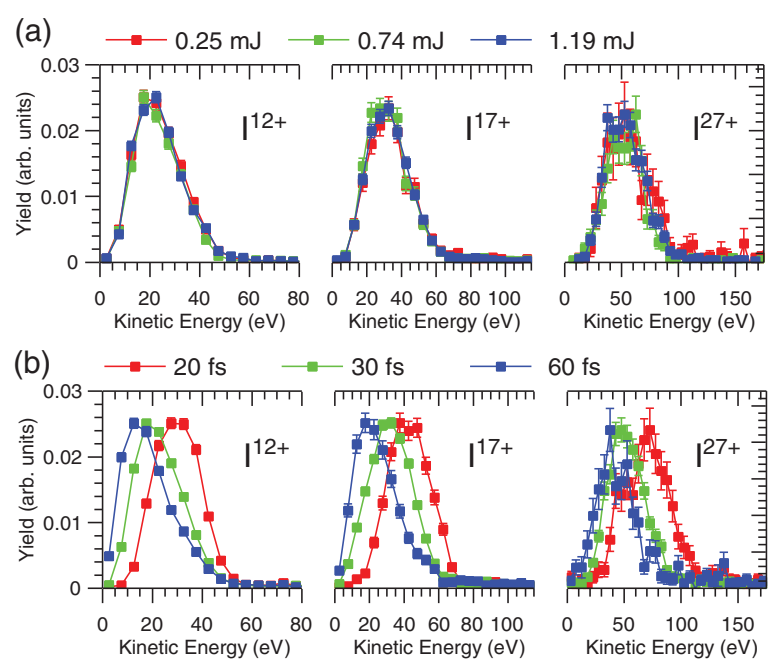

FIG. 1. Representative experimental iodine ion kinetic energy distributions for (a) 30-fs x-ray pulses with different pulse energies, and (b) for $0.37 \mathrm{~mJ}$ x-ray pulses with different pulse durations.

illustrated in Fig. 2, where the measured and calculated average charges of carbon ions detected in coincidence with a given iodine charge state are shown. In both experiment and simulation, the average carbon charge for a given iodine charge state with more than six charges is smaller for longer pulses. Since the carbon fragments are almost exclusively ionized via electron transfer to the iodine ion, the lower carbon ion charge suggests that the electron rearrangement is less efficient for longer pulses. Qualitatively, this can be expected, because for longer pulses a given total charge state is, on average, reached at larger internuclear separations, and charge transfer probability decreases with increasing distance [36]. To better understand the difference in the charge transfer, in Fig. 3 we calculated the average time evolution of the charges and the iodine-carbon distance for those interactions which end up with an iodine ion charge of $20+$. According to the classical over-the-barrier model $[18,37,38]$, the critical internuclear distance, above which the charge transfer from $\mathrm{C}^{3+}$ to $\mathrm{I}^{21+}$ is forbidden, is $4.98 \AA$ [black dashed line in Fig. 3(b)]. For a pulse duration of $10 \mathrm{fs}$, the C-I distance at which the carbon ion acquires a charge of $3+$ is about 3.97 A, i.e., smaller than the critical distance. For a $60 \mathrm{fs}$ pulse, on the other hand, the C-I distance is already much larger than the critical distance before the carbon ion reaches a charge of 3+ [black dashed line in Fig. 3(a)]. These observations agree with the pulse duration dependence of charge transfer observed in Fig. 2.

As can be seen from Fig. 2, the calculated average carbon charge for the longest pulse (60 fs) is suppressed more than its experimental value. A possible reason for this discrepancy can originate from shortcomings of our molecular electronic structure model at large internuclear distances, at which the probability of charge rearrangement sensitively depends, e.g., on the employed basis set. 


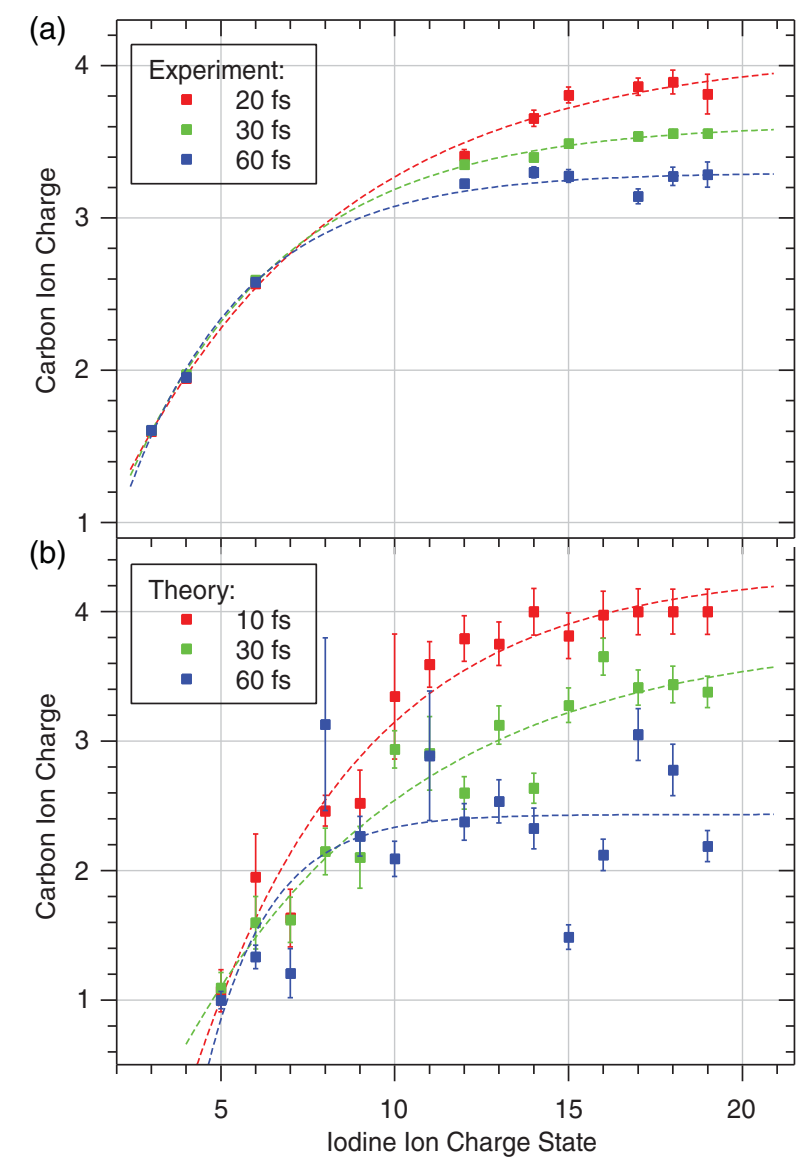

FIG. 2. Measured (a) and calculated (b) charge of carbon ions detected in coincidence with a given iodine ion charge state for different pulse durations with $0.37 \mathrm{~mJ}$ pulse energy. The vertical bars show the standard errors of the carbon ion CSDs. Dashed lines are empirical fits to guide the eye (see SM).

The comparison of the measured iodine ion CSDs for different pulse durations is displayed in Fig. 4(a). Overall, similar ion charge state distributions are observed, as can be expected for a sequential ionization process if the pulse energy is kept constant. However, apart from this overall similarity, the experimental data show a systematic pulse duration dependence beyond the statistical error for charge states above $\mathrm{I}^{18+}$. In this region, shorter pulses result in a higher yield, with up to a factor of 2 increase between 20 and $60 \mathrm{fs}$ for charge states above $\mathrm{I}^{25+}$. While the increase of high charge state production for shorter pulses at a given pulse energy can be expected if direct ("simultaneous") multiphoton absorption plays an important role [39], for our experimental conditions the contribution from such direct processes, estimated following the procedure described in [40], is expected to be approximately 2500 times smaller than from the dominant mechanism of sequential multiphoton ionization. Although it was recently predicted theoretically $[41,42]$ that at ultrahigh intensities, above $10^{20} \mathrm{~W} / \mathrm{cm}^{2}$, sequential multiphoton ionization starts to mimic the quasinonsequential behavior, the current

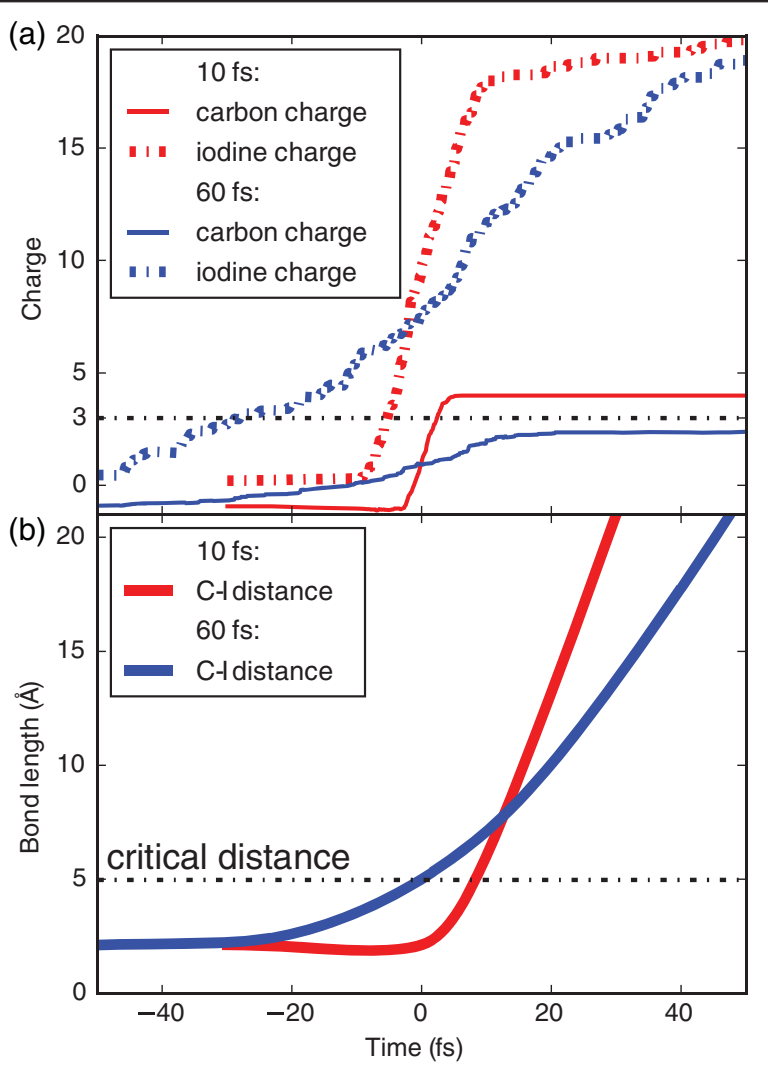

FIG. 3. Average charge evolution (a) and internuclear distance (b) of carbon and iodine for those interaction events ending up with iodine ion charge of $20+$, and for pulse durations $10 \mathrm{fs}$ and 60 fs. Note that (a) and (b) share the same $x$-axis. The black dashed line in (a) marks an ion charge of $3+$, while the dashed line in (b) is drawn at the critical distance $(4.98 \AA)$ for the charge transfer from $\mathrm{C}^{3+}$ to $\mathrm{I}^{21+}$. The time axis is defined such that the peak of the XFEL pulse is at $0 \mathrm{fs}$.

experiment used much lower intensities and is still safely within the sequential ionization regime. For sequential $\mathrm{x}$-ray ionization, however, the opposite trend, in which the yield of the highest charge states is higher for longer pulses, has been reported previously, and was explained by the non-negligible "refilling" time of the created innershell vacancies-an effect dubbed "intensity-induced transparency" or "frustrated absorption" [7,10,12,29].

From our previous study [16], we know that iodine ions with charge states above $\mathrm{I}^{25+}$ are almost exclusively detected in coincidence with $\mathrm{C}^{4+}$. In addition, the results shown in Figs. 2 and 3 reveal that $\mathrm{C}^{4+}$ ions mainly appear in coincidence with high iodine charge states, and more $\mathrm{C}^{4+}$ ions are produced with shorter pulses due to easier charge transfer. The combination of these findings is consistent with the observation of the increased production of highly charged iodine ions for shorter pulses observed in Fig. 4(a). Qualitatively, we attribute such an observation to ionization enhancement by charge transfer. In the original CREXIM model [16,24], the level of molecular ionization enhancement was directly determined by the number of 


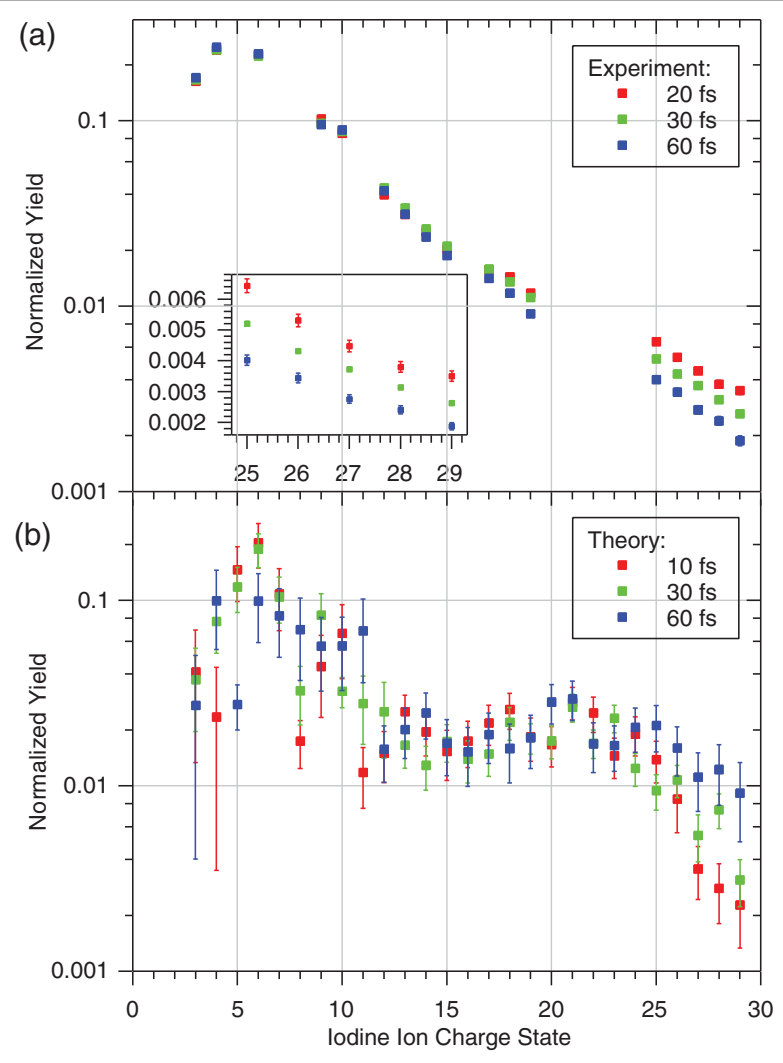

FIG. 4. Iodine ion charge state distributions for different pulse durations at $0.37 \mathrm{~mJ}$ pulse energy. The inset in the top figure shows the yield of high charge states on a linear scale. Each distribution is normalized such that the total yield equals 1 .

electrons transferred to the absorbing heavy atom from the molecular partners. Within this picture, the charge rearrangement increases the total charge state of the molecule but cannot increase the charge state of the heavy fragment (iodine). However, electron transfer to the iodine site can have further consequences: When an electron is transferred to the iodine valence shell, it influences the timing and energetics of Auger decay processes, e.g., facilitating certain Auger pathways and enabling new ones. These effects, which we dub "extended CREXIM", can result in the observed higher level of ionization of the iodine atom for shorter pulses, for which the charge rearrangement is more efficient.

As illustrated in Fig. 4(b), this overall trend of the pulse duration dependence is not reproduced by the XMOLECULE calculation, indicating the need to further improve the theoretical modelling in order to describe the subtleties of charge-transfer dynamics and their effect on the CSDs. While we assume that the disagreement originates from a combination of several effects not included in the current version of XMOLECULE (which are discussed in more detail in SM), its exact reasons remain unclear. A more definite and quantitative explanation of the observed behavior would require further elaboration of the model, along with a more precise experimental characterization of the spatiotemporal profile of the x-ray pulse.

In summary, we have presented a detailed experimental and theoretical analysis of the ultraintense hard x-ray ionization and fragmentation of a prototypical heteronuclear molecule, focusing on the dependence on the pulse energy and pulse duration. We show that the kinetic energies of the resulting ionic fragments strongly depend on pulse duration, but not on pulse energy. This behavior is determined by the average time interval between the sequential photoabsorption steps leading to the creation of a particular ion charge state, which increases with the pulse duration but is independent of the pulse energy. Experimentally, shorter pulses produce more ions with high charge states, which we attribute to more efficient charge rearrangement. Our experimental results provide benchmark data for a detailed comparison with state-of-the-art calculations and point out potential limitations of the theoretical model used. The observed trends are likely applicable to other molecular systems in the regime where the ionization dynamics is largely defined by charge transfer and interatomic relaxation processes. They also should be taken into account when choosing pulse parameters and modelling the outcome of other XFEL experiments such as coherent imaging [43-45].

This work is supported by the US Department of Energy, Office of Science, Basic Energy Sciences, Chemical Sciences, Geosciences, and Biosciences Division, who supported the Kansas group under Contract No. DEFG02-86ER13491 and the Argonne group under Contract No. DE-AC02-06CH11357. X. L. L.I. and R. S. were partially supported by the Award No. DE-SC0019451 from the same agency. Theoretical work was supported in part by the Cluster of Excellence 'The Hamburg Centre for Ultrafast Imaging' of the Deutsche Forschungsgemeinschaft (DFG)—EXC 1074-Project ID 194651731. Use of the Linac Coherent Light Source (LCLS), SLAC National Accelerator Laboratory, is supported by the US Department of Energy, Office of Science, Office of Basic Energy Sciences under Contract No. DE-AC02-76SF00515. T. G. acknowledges the Peter-Ewald Fellowship from the Volkswagen Foundation. K. U. acknowledges the XFEL strategy program of MEXT, the Dynamic Alliance for Open Innovation Bridging Human, Environment and Materials, and the TAGEN project for support. We are grateful to the SLAC staff for their support and hospitality during the beamtime, and to Kevin D. Carnes for carefully reading the manuscript.

[1] C. Bostedt, S. Boutet, D. M. Fritz, Z. Huang, H. J. Lee, H. T. Lemke, A. Robert, W. F. Schlotter, J. J. Turner, and G. J. Williams, Rev. Mod. Phys. 88, 015007 (2016).

[2] L. Young et al., J. Phys. B 51, 032003 (2018).

[3] H. N. Chapman, Annu. Rev. Biochem. 88, 35 (2019). 
[4] R. Neutze, R. Wouts, D. van der Spoel, E. Weckert, and J. Hajdu, Nature (London) 406, 752 (2000).

[5] H. N. Chapman et al., Nature (London) 470, 73 (2011).

[6] T. Ekeberg, M. Svenda, C. Abergel, F. R. N. C. Maia, V. Seltzer et al., Phys. Rev. Lett. 114, 098102 (2015).

[7] L. Young et al., Nature (London) 466, 56 (2010).

[8] B. Rudek et al., Nat. Photonics 6, 858 (2012).

[9] H. Fukuzawa, S.-K. Son, K. Motomura, S. Mondal, K. Nagaya et al., Phys. Rev. Lett. 110, 173005 (2013).

[10] P. J. Ho, C. Bostedt, S. Schorb, and L. Young, Phys. Rev. Lett. 113, 253001 (2014).

[11] B. Rudek et al., Nat. Commun. 9, 4200 (2018).

[12] L. Fang et al., Phys. Rev. Lett. 109, 263001 (2012).

[13] B. Erk, D. Rolles, L. Foucar, B. Rudek, S. W. Epp et al., Phys. Rev. Lett. 110, 053003 (2013).

[14] B. Erk et al., J. Phys. B 46, 164031 (2013).

[15] K. Motomura et al., J. Phys. Chem. Lett. 6, 2944 (2015).

[16] A. Rudenko et al., Nature (London) 546, 129 (2017).

[17] K. Mertens, N. Gerken, S. Klumpp, M. Braune, and M. Martins, J. Mod. Opt. 63, 383 (2016).

[18] B. Erk et al., Science 345, 288 (2014).

[19] R. Boll et al., Struct. Dyn. 3, 043207 (2016).

[20] L. Fang, H. Xiong, E. Kukk, and N. Berrah, Appl. Sci. 7, 529 (2017).

[21] M. Hollstein et al., New J. Phys. 21, 033017 (2019).

[22] K. Nagaya, K. Motomura, E. Kukk, H. Fukuzawa, S. Wada et al., Phys. Rev. X 6, 021035 (2016).

[23] T. Takanashi et al., Phys. Chem. Chem. Phys. 19, 19707 (2017).

[24] L. Inhester, K. Hanasaki, Y. Hao, S.-K. Son, and R. Santra, Phys. Rev. A 94, 023422 (2016).

[25] K. Nass et al., J. Synchrotron Radiat. 22, 225 (2015).

[26] L. Galli et al., Struct. Dyn. 2, 041703 (2015).

[27] S.-K. Son, H. N. Chapman, and R. Santra, Phys. Rev. Lett. 107, 218102 (2011).
[28] L. Galli et al., IUCrJ 2, 627 (2015).

[29] M. Hoener, L. Fang, O. Kornilov, O. Gessner, S. T. Pratt et al. Phys. Rev. Lett. 104, 253002 (2010).

[30] S. Boutet and G. J. Williams, New J. Phys. 12, 035024 (2010).

[31] See Supplemental Material at http://link.aps.org/ supplemental/10.1103/PhysRevLett.127.093202 for details of the pulse energy filtering, pulse duration determination, and for additional simulations, which also includes an additional Ref. [32].

[32] C. Behrens et al., Nat. Commun. 5, 3762 (2014).

[33] Y. Hao, L. Inhester, K. Hanasaki, S.-K. Son, and R. Santra, Struct. Dyn. 2, 041707 (2015).

[34] K. Toyota, Z. Jurek, S.-K. Son, H. Fukuzawa, K. Ueda, N. Berrah, B. Rudek, D. Rolles, A. Rudenko, and R. Santra, J. Synchrotron Radiat. 26, 1017 (2019).

[35] X. Li, R. Boll, D. Rolles, and A. Rudenko, arXiv:2012 .06632 .

[36] V. May and O. Kühn, Charge and Energy Transfer Dynamics in Molecular Systems (Wiley-VCH, Weinheim, 2011).

[37] H. Ryufuku, K. Sasaki, and T. Watanabe, Phys. Rev. A 21, 745 (1980).

[38] A. Niehaus, J. Phys. B 19, 2925 (1986).

[39] N. Gerken, S. Klumpp, A. A. Sorokin, K. Tiedtke, M. Richter, V. Bürk, K. Mertens, P. Juranić, and M. Martins, Phys. Rev. Lett. 112, 213002 (2014).

[40] G. Doumy, C. Roedig, S.-K. Son, C. I. Blaga, A. D. DiChiara et al., Phys. Rev. Lett. 106, 083002 (2011).

[41] S.-K. Son, R. Boll, and R. Santra, Phys. Rev. Research 2, 023053 (2020).

[42] P. J. Ho, E. P. Kanter, and L. Young, Phys. Rev. A 92, 063430 (2015).

[43] O. Y. Gorobtsov, U. Lorenz, N. M. Kabachnik, and I. A. Vartanyants, Phys. Rev. E 91, 062712 (2015).

[44] P. J. Ho, C. Knight, M. Tegze, G. Faigel, C. Bostedt, and L. Young, Phys. Rev. A 94, 063823 (2016).

[45] C. H. Yoon et al., Sci. Rep. 6, 24791 (2016). 